\title{
Design and Fabrication of Automated Inbuilt Hydraulic Jack for Light Motor Vehicle
}

\author{
Mohan Kumar R S, Vinayagamoorthi M A
}

\begin{abstract}
In the feat of changing the wheel of a light motor vehicle, lifting the vehicle is an unavoidable and a complicated practice. Manual lifting using jack systems requires more human effort and comparatively time consuming also. The proposed model is an alternative solution which reduces both the human effort and vehicle lifting time. To lift the vehicle, the user need to exert a gentle push over the lever, that actuates the desired hydraulic jack to the preferred height. In various proposals carried earlier regarding lifting the vehicle more number of jack systems, various power source terminals and requires an additional actuating means, which is redesigned with optimized conditions. The proposed system increases the comfort of the light motor vehicle users which brings them more closer to the technological up gradation. The proposed project is also mainly concentrated towards the ease of differently abled and aged persons.
\end{abstract}

Keywords - Hydraulic jack, Light motor vehicle, Redesign, Lifting force

\section{INTRODUCTION}

The proposed system is a comfort based functional system which is used to lift the vehicle without any difficulty, experience and physical work. The system is mainly built valuing the difficulties experienced by the elderly persons and physically challenged persons. The main lead of the proposed system is the number of jacks used is constrained; the power for the actuation is directly obtained from the engine of the vehicle. The direct power extraction leads to the elimination of the battery and the motor, which significantly grades the reduction of weight and the cost. This enhances the affordability of the system.

\section{LITERATURE SURVEY}

The conceptual thought of lifting the vehicle using jacks has emerged earlier at the period of late 1920's [1]. Pneumatic jacks were initially prescribed for this scenario since it can be compressed; it was believed that it had a far better scope than any other working fluid in nature to lift the vehicle. But as time emerged it is found that the better and efficient means of lifting vehicle was hydraulics which is still in practice in lifting the heavier loads, since hydraulics are provided with accumulators so the rate of getting injured due to failure is considerably reduced. Usually a jack is consisted of a cylinder and a piston in most of the cases the guiding medium is stationary and the sliding piston ${ }^{[1]}$ is restricted in it which would result in the uplifting of the

Revised Manuscript Received on August 14, 2019.

Mohan Kumar R S, Assistant Professor, Department of Mechanical Engg, Kumaraguru College of Technology, Coimbatore, India. (E-mail: mohankumar.rs.mec@kct.ac.in)

Vinayagamoorthi M A, Assistant Professor, Department of Mechanical Engg, Kumaraguru College of Technology, Coimbatore, India. (E-mail: vinayagamoorthi.ma.mec@kct.ac.in) components connected to those structures. There are many other methods to constrain the cylinder piston arrangement and even their execution is carried out using many numbers of methods. When a novel hydraulic system is provided for the vehicle jacks whereby the various jacks attached to a vehicle can be quickly and easily controlled ${ }^{[2]}$ from a common central point and one or more of the jacks may be extended or collapsed as desired.

Regarding assembly and positioning of the cylinders there are various methods that are being implemented, placing the cylinder in both the front and back of the car ${ }^{[3]}$ it requires a more accurate and a precise design comparatively because it is placed beneath the engine which comprises a lot of difficulties within for space requirements and mass balance. The other notable method is using hydraulic jacks in all the four wheels of the car to lift the car ${ }^{[2]}$ when need arises. But this method again adds even more complications by adding weight to the car and also it is not cost effective because the fabrication of each single cylinder is an extra burden on their back for both the manufacturer and the consumer, the manufacturer will not be able to hike his cost over a certain level either the consumer will not be affordable enough to buy that particular product which is so expensive.

The disadvantages and defects of the models mentioned above in this chapter are discussed in the following passages. First the model which has the hydraulic jack in the front and rear end ${ }^{[3]}$ of the car is discussed. For the efficient placement and for achieving better mass balance effects ${ }^{[13]}$ the engine assembly has to be rearranged and the placement of the cylinders are to be done if not the available space should be used efficiently which is not possible in practice. Other disadvantages are the mass balance feature this is achieved only when the placement axis attains symmetric nature, if not then the mass balance will not be up to the expected level which will lead to the wobbling of the vehicle at once when it gets lifted. The actuation is also a tougher task because the cylinder in front of the car which would experience more loads than the rear because of the weight of the engine so the cylinders has to be manufactured for two different specifications, if it was for same specifications then the rear cylinder would have more lifting force than required. This particular function will demand more oil or the working fluid which would ultimately increase the capacity of the reservoir tank and the weight of the whole setup. The setup requires more clearance from the ground level since lifting it from the mid plane of the car which is also a tedious action to perform and achieve the required result. 


\section{DESIGN AND FABRICATION OF AUTOMATED INBUILT HYDRAULIC JACK FOR LIGHT MOTOR VEHICLE}

The model which is prescribed with four cylinders for lifting ${ }^{[6]}$ the vehicles is one of the leading conceptual thought in trend but it too has its own demerits. The placement of the cylinders should be made at the axle of all the four wheels which itself is a difficult process. The usage of four cylinders doubles the weight of the setup which uses two cylinders which is also an undesirable proposal ${ }^{[14]}$ for the user. The weight of the cylinder will act on the axle of the wheel continuously which is not built in the way to sustain the additional pressure constantly ${ }^{[7]}$; this may lead to deformation of the material. The cylinders working fluid storage tank will become larger since it should provide the working fluid for all the four cylinders. The noted disadvantage is this setup requires a motor to run the pump in the circuit and the motor requires a power supplying source, in most of the proposed models the battery is chosen as a power supplying source which alone adds up nearly 90 kilograms of weight to the setup. This weight addition will lead to the mileage drop and which would then remain in the market as an unsold stuff which reduces the efficiency of the vehicle.

The other major model which is being proposed over to the automobile industries are the hydraulic jack which will be fixed in the chassis or axle of the car and during the period of execution of the cylinder the fluid is taken from the reservoir which supplies working fluid for the brakes and steering ${ }^{[2]}$. The main disadvantage is the extraction of the fluid from the reservoir which is supplying working fluid to the brakes and steering, because both are very vital parts of the vehicle whose failure would lead to serious effects. Leakage in the circuit may lead to hazardous action. The circuit becomes more complicated than the others comparatively and the rectification of errors and repairing the assembly components may be difficult.

The methodology which is proposed by us is a far better approach which will be able to provide remedies for most of the problems mentioned above. The number of cylinders used in the proposed method is just two which would greatly influence the cost of the entire unit. The working fluid is required for just two cylinders which would result in the lesser storage ${ }^{[7]}$ of hydraulic fluid; it ultimately reduces the size of the reservoir tank and also reduces the weight of the circuit. The other main advantage of the proposed setup is it does not require a motor to run the pump in the circuit. The power for the pump is directly extracted from the engine which eliminates the need of the battery. Since there is no battery the weight of the circuit is again reduced to a considerable level. The proposed method also incurs an advanced direction control valve which cuts down the complication of the circuit and simplifies the circuit. Repair and maintenance of the circuit or the assembly components will be easier, comparatively.

The other major advantages are the placement position and the symmetric nature of the lifting process. The mass balance is achieved by placing the cylinder and lifting it in such a way that the base of the piston and the other two wheels in the opposite of that particular cylinder is in the ground, which provides a triangular support to the vehicle which would result in the better and stable lifting of the car.

\section{PROBLEM DEFINITION}

Lifting the vehicle using conventional method requires a lot of manual work and is a tedious process; it demands a new methodology which is to be implemented that eradicates all the existing problems. The proposed system enhances the ease lifting the vehicle and eliminates the existing problems. The system adopts itself and delivers optimum performance irrespective of the landscape, climatic conditions and other factors. It does not insist further complications and unique arrangement in the existing product.

Usage of more number of hydraulic jacks for each vehicle increase the weight of the entire system which is not desirable. Using a motor for a pump is also a tedious process because the motor needs a battery to supply power to the motor which again increases the weight of the system to a considerable extent which would deteriorate the efficiency of the vehicle. So using optimum design and inculcating only the exact requirements, the proposed system is fabricated. This eliminates the difficulties faced by the earlier proposals.

\section{PROPOSED METHODOLOGY RESULTS}

A separate system consisting of two hydraulic jacks, pump and a control valve will be connected and positioned in the vehicle. The pump will be coupled with the main spindle shaft of the engine which has the unchanged rotations of the engine crank. The shaft of the pulley and the engine spindle is coupled using a distinctively designed belt drive. The idle conditions results in the flow of the hydraulic fluid from the reservoir to the reservoir without doing any work, so that the energy from the engine is not consumed. When there arises a need for lifting the vehicle the driver needs to exert a gentle push to the actuating lever provided up to the extent of the height required. Thus when he actuates the lever the directional control valve directs the hydraulic fluid to the required hydraulic jack and results in the actuation process. The retraction phase of the hydraulic jack is also very similar to the actuation process but in contrast the lever has to be pushed in the opposite direction to the earlier. Thus the pump develops a pressure up to 100 bars within the limited time interval and the load conditions exerted in the vehicle. The hydraulic jack is designed considering the aspects of various considerations and fabricated in the mode that it could lift about two tonnes individually. 


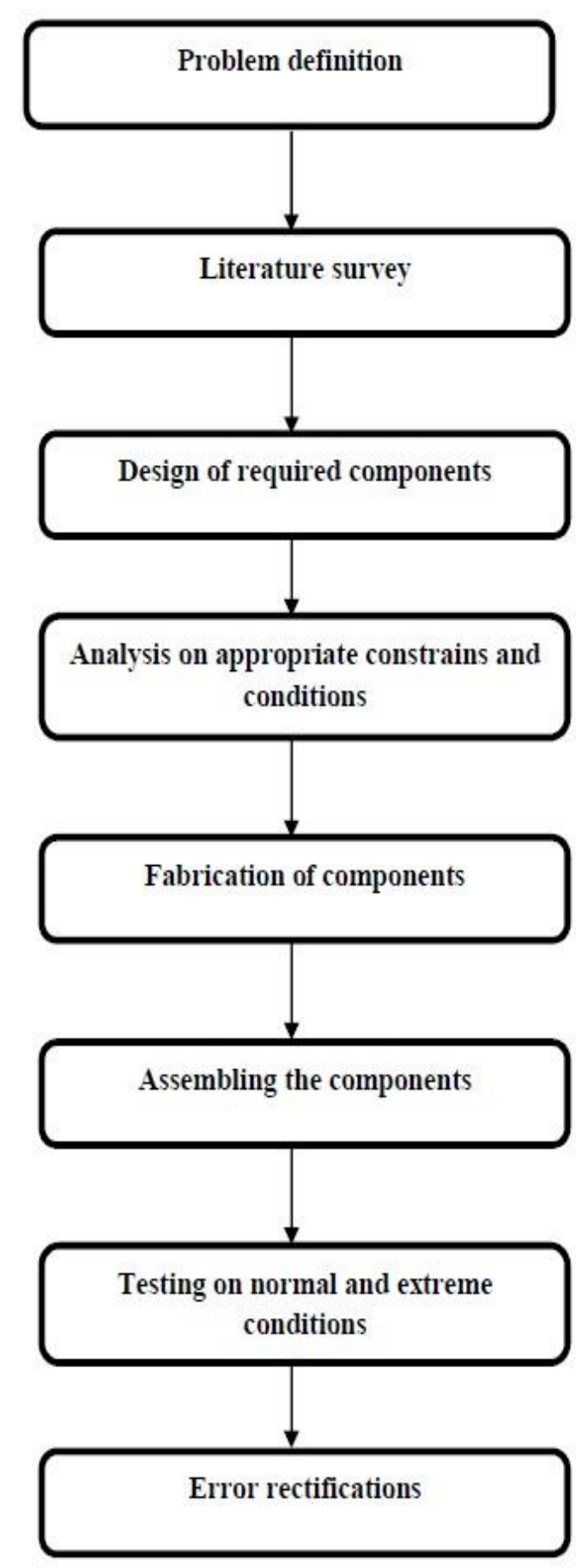

Fig 1: Proposed methodology

\section{DESIGN CALCULATIONS \& RESULTS}

\subsection{Design of hydraulic jack}

The design of the hydraulic jack includes the following consideration which includes,

- Positioning features,

- Overall dimensions,

- Volume of space required,

- Weight exerted and the changes likely to be made.

Cylinder Diameter $(\mathrm{d})=50 \mathrm{~mm}$

$$
\begin{aligned}
\text { Area of the cylinder } & =\frac{\pi d^{2}}{4} \\
& =\frac{\pi x 50^{2}}{4} \\
& =1963.495 \mathrm{~mm}^{2} .
\end{aligned}
$$

The lifting force required for the normal vehicles would be nearly 600 kilograms since there are two hydraulic jacks placed symmetrically at either side of the vehicle Considering sports utility vehicles (SUV), multi utility vehicle (MUV), and in case of physically challenged some of the passengers might find it difficult to step down. Inculcating all these above factors a comfort range of two tonne lifting force is provided for each cylinder. Thus the calculations for the lifting force of the hydraulic jack is as follows,

$$
\begin{aligned}
\text { Lifting Force } & =2000 \mathrm{~kg} \\
& =2000 \mathrm{~kg} * 9.81 \\
& =19620 \mathrm{~N}
\end{aligned}
$$

For the designed diameter of the cylinder and the total height to be lifted to isolate the wheels from the ground and the energy from the engine output in considered in this pressure calculations and in the selection and implementation of the pump. Thus the theoretical calculations of the pressure signifies that the pressure of about 100 bar is required, which is generated by the compact gear pump which is implemented in the system.

Pressure acting on the cylinder $(\mathrm{p})=$ Lifting force $/$ Area of the cylinder

$$
\begin{aligned}
& =19620 / 1963.495 \\
& =9.9923 \mathrm{~N} / \mathrm{mm}^{2} \\
& =99.923 \mathrm{bar} \\
& \approx 100 \mathrm{bar}
\end{aligned}
$$

Stroke Length of the cylinder $=279.4 \mathrm{~mm}$.

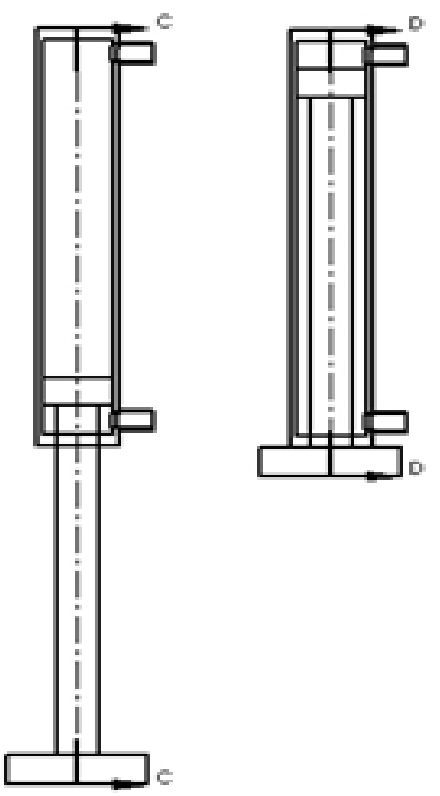

Fig.2 Design of hydraulic jack

\subsection{Design of pulley}

Pulley $\mathrm{P} 1$ = The driver pulley

Pulley P2= The driven pulley 


\section{DESIGN AND FABRICATION OF AUTOMATED INBUILT HYDRAULIC JACK FOR LIGHT MOTOR VEHICLE}

Two pulleys have to be designed as per the requirement of the engine and the type of vehicle that is in use. The pulleys are to be designed in such a way that the Pulley P2 connected in the shaft of the pump should step down the energy that is transmitted from the Pulley P1 which is coupled with the main spindle shaft of the engine.

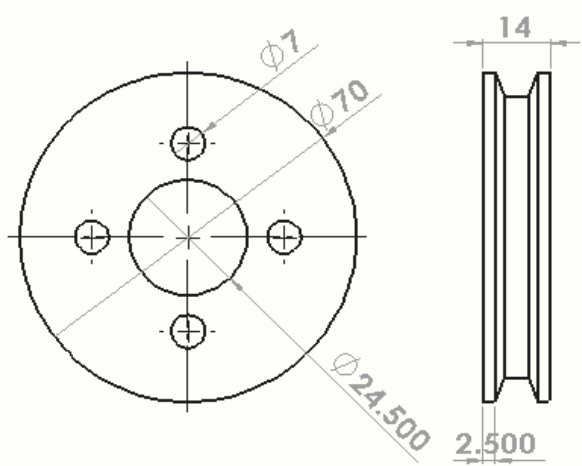

Fig.3 Design of pulley P1

The design of Pulley P1 shown in fig. 3 includes the design considerations of the existing pulley that is present in the engine which is used to run the radiator fan and the

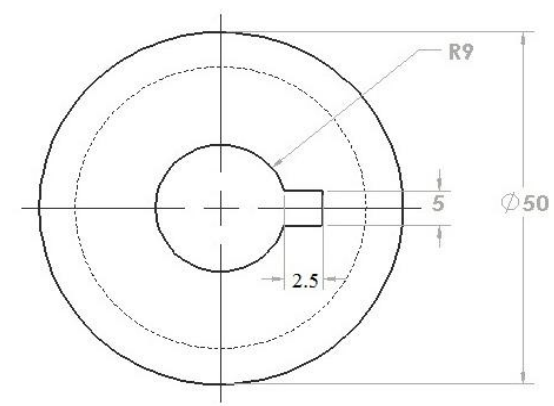

Fig.4 Design of pulley P2

The design of Pulley P2 shown in fig.4 includes the design of the Pulley P1 and the ratio at which the power is to be stepped down. The design should be made and confirmed that the energy obtained in the form of rotations per minute (RPM) should be within the prescribed limit of the pump used. Exceeding the limit might result in adverse effects.

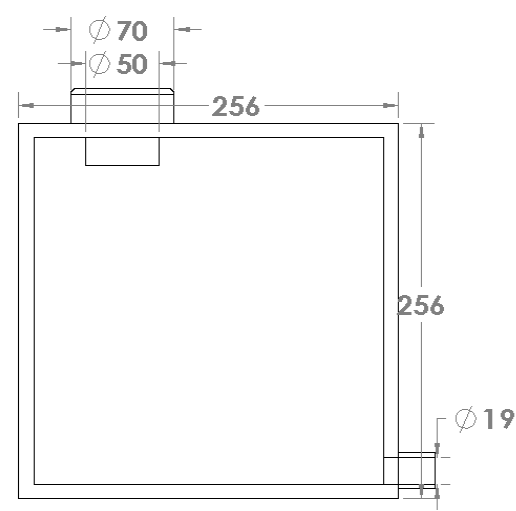

Fig.5 Design of Reservoir

5.3. Design of reservoir tank coolant pump.

The reservoir shown in fig. 4 is designed and fabricated in a manner that it can hold the exact amount of fluid required, to avoid the fluid tumbling to and fro inside the reservoir tank and to reduce the addition of weight. The reservoir is fabricated and firmly fixed using fixtures under the rear seat of the vehicle.

\subsection{Design of belt drive}

Driven pulley diameter:

Rpm of Driver pulley $=900$

Rpm of Driver pulley $=1300$

Dia of driver pulley $=70 \mathrm{~mm}$

Dia of driven pulley $=900 / 1300 * 70$

$$
=48.46 \mathrm{~mm}
$$$$
=50 \mathrm{~mm} \text {. }
$$

Belt Length:

$$
\begin{aligned}
& =2 \mathrm{C}+1.57(\mathrm{D}+\mathrm{d})+\frac{(\mathrm{D}-\mathrm{d})^{2}}{4 \mathrm{C}} \\
& =2 * 600+1.57(70+50)+\frac{(50-70)^{2}}{4 * 600} \\
& =1390 \mathrm{~mm}
\end{aligned}
$$

Centre to centre distance $=600 \mathrm{~mm}$

Speed Ratio:

Dia of Driven/Dia of Driver $=50 / 70$

$=0.72: 1$

Belt velocity

$$
\begin{aligned}
& \mathrm{V}=\frac{\pi * \mathrm{D} * \mathrm{~N}}{60} \\
& =\frac{\pi * 0.07 * 900}{60} \\
& =3.32 \mathrm{~m} / \mathrm{s}
\end{aligned}
$$

Angle of arc of contact:

$$
\begin{aligned}
& \theta=180-2 \sin -1[(\mathrm{D}-\mathrm{d}) / 2 \mathrm{C}] \\
& =180-2 \sin -1[(70-50) / 2 * 600] \\
& =178.04^{\circ}
\end{aligned}
$$

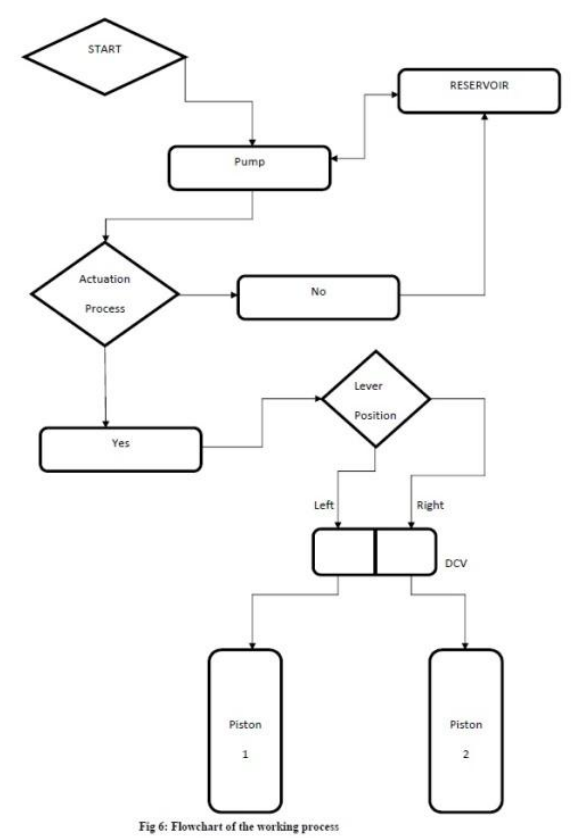




\section{CONCLUSION}

The proposed system is designed, fabricated and implemented as a real time project. All the proposed methodologies and the suggested ideas have been properly analysed implemented and has been attempted in a real time engine. The system is designed and fabricated in the aspect that it is more likely to be commercialized and also fulfils the problems that is taken in to account. The proposed system has its own advantages and is a result in the best affordable choices available. It can be upgraded and used even in the heavier vehicles with slighter design modifications and positioning features.

\section{REFERENCES}

1. Clarence E. Williams of Kansas University, Missouri, "Pneumatic jack", USPO patent paper, serial number 1957151.

2. M.D. Hall, "Hydraulic system for vehicle lifts", USPO patent paper, serial number 2472294.

3. Vincent R Paolucci, "Hydraulic brake and jack combination unit control device for vehicle", USPO patent paper, serial number 2764249.

4. Paul E Hanser, Meier, Vanroekel, "Automatic levelling system”, USPO patent paper, serial number 4746133.

5. C.E.Williams, "Pneumatic jack", USPO patent paper, serial number 171940.

6. "Machine design", Sundararajamoorthy T.V, Shanmugam.N, Anuradha publications, 2003.

7. "AUTOMOTIVE MECHANICS", S.Srinivasan, McGrawHill Education publications, Edition-2006, ISBN 0070966303.

8. "AUTOMOBILE ENGINEERING", Dr.K.R.Govindan, Edition-2004.

9. “THE AUTOMOBILE”, Harbans Singh Reyat, S. chand \& Company ltd publications Edition-2009.

10. "Handbook of Rigging: For Construction and Industrial Operations", MacDonald, Joseph A. McGraw-Hill Professional publications.

11. "Kinematics and Dynamics of Planar Machinery",B. Paul, Prentice-Hall publications, 1979 Edition.

12. John A. Jakle, Keith A. Sculle. (2004). Lots of Parking: Land Use in a Car Culture. Charlottesville:article.

13. "Classical Dynamics of Systems and Particles", Marion, JB; Thornton, ST (1995). Edition 4, Thomson publications..

14. "AUTOMOTIVE TECHNOLOGY", H.M.Senthil, Edition2003.

15. "PSG design data book", revised edition of 1978, reprinted on 2011. 